\title{
The effects of process parameters on forming limit of rectangular tube in rotary draw bending process
}

\author{
Kuanxin Liu \\ Ningbo Branch of China Academy of Ordance Science, Ningbo 315103, China \\ Northwestern Polytechnical University \\ Shunqi Zheng \& Gang Chen \\ Ningbo Branch of China Academy of Ordance Science, Ningbo 315103, China
}

\begin{abstract}
In the manuscript, the forming limit for cross-section distortion is defined according to the employing area and aviation standard. First, the definition and determination method of forming limits is proposed. And then, the effects of process parameters on forming limit are investigated based the 3D FE simulation model. Finally, the 3D forming limit diagrams for cross-section distortion are obtained under different process parameters.
\end{abstract}

KEYWORD: Forming limit; determination method; Forming limit diagram

\section{INTRODUCTION}

Thin-walled rectangular aluminum alloy tube possesses a combination of light weight, high stiffness, strong vibration, large volume, low loss of waveguide, which has attracted many applications in the aerospace, aviation, automobile and radar. Among various bending methods, such as pure bending, stretch bending, press bending and rolling bending, rotary draw bending is nowadays the most universal approach to produce this type of thin-walled bent tubes in virtue of high efficiency and high precision [1-2]. The cross-section distortion is inevitably avoided during the bending process, and it may cause severe damage to tools and machines. The cross-section distortion, which contains not only the concave of the flange but also the convex of the web, directly weakens the mechanical behaviors and the ability of signal wave transmission. In the rotary draw bending process, the cross-section distortion seriously influences the quality of rectangular waveguide and weakens the resistance to instability, inducing the larger loss wave ratio. The cross-section distortion is the vital factor determining forming limit with the demand of higher dimension precision and smaller bending radius. It is of great significance to predict and control the forming limit.

In the last decades, many scholars have investigated the cross-section distortion and forming limit in tube bending process by experiment, theoretical and FE simulated methods. In Northwestern polytechnical university, many scholars have investigated the forming characters of tube bending [3-6]. A wrinkling prediction analytical model is established to derive the minimum bending radius, and the zonal distribution of forming limit for wrinkling is drawn by Yang[7,8]. Zhao[9] investigates the wrinkling forming limit in rotary draw bending, and obtains the forming limit diagrams. The energy prediction model for wrinkling is obtained by Yan[10] based on the FE simulation model and minimum energy principle. The prediction model employing the CDM and GTN model can predict the forming limit for crack in 50520 aluminum alloy tube bending[11]. The experiments are carried out by Okude[12] combined with FE simulation in order to study the forming limit of aluminum alloy tube bending, it is found that the axial force can raise the bending level. These investigations and achievements are of importance and significance to establish the FE model and to analyze the forming limit forming in tube bending for cross-section distortion in rectangular tube rotary draw bending process.

\section{THE EFFECT OF OF PROCESS PARAMETERS ON FORMING LIMIT}

\subsection{Establishment of FE model.}

With the requirements of higher dimensional intolerance and smaller bending radius, the optimal process parameters and minimum bending radius should be determined. According to the standard and the wave loss ratio, the cross-section distortion not exceeding $10 \%$ is defined to be the forming limit, and the minimum bending radius with forming limit is defined to be minimum bending radius. Taking the 3A21 aluminium alloy rectangular tube with dimen- 
sions $24.86^{*} 12.2 * 1 \mathrm{~mm}\left(b^{*} h^{*} t\right)$ as an example, a three dimensional finite elements model of this process, as shown in Fig.2, is built under the ABAQUS/explicit environment based on the solution of several key techniques, such as model creation, contact boundary condition treating, material properties definition, meshing technology, and it is validated to be credible [9].

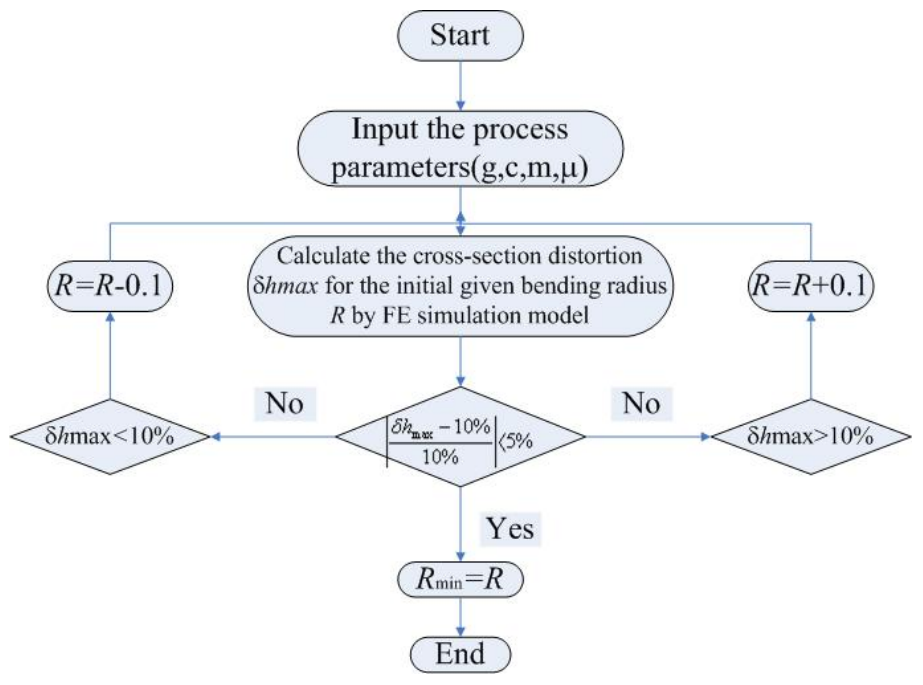

Fig.1Determination method of forming limit

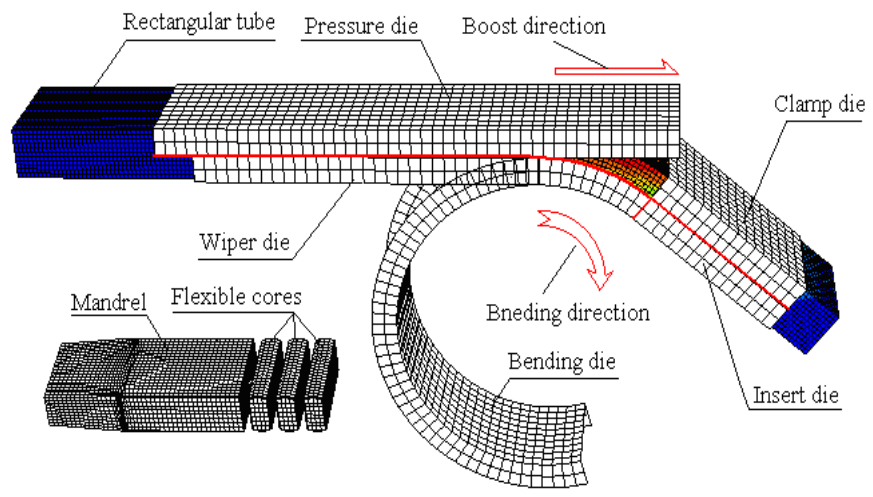

Fig.2 3D FE simulation model

\subsection{The effects of material parameters on forming limit}

\subsubsection{The effects of elastic modulus.}

The elastic modulus $E$ is set in the range 40 200GPa respectively, and the curves of minimum bending radius vs elastic modulus are shown in Fig.3.

It can be seen that the minimum bending radius increases from 30 to $40 \mathrm{~mm}$ with elastic modulus increasing from 40 to $200 \mathrm{MPa}$. The elastic modulus is the parameter to measure the ability to resist the elastic deform, while the plastic deform is the main deform. Larger elastic modulus will need larger force imposed by dies, inducing larger elastic springback. Therefore, the effects of elastic modulus on forming limit is not significant.

\subsubsection{The effects of strengthen factor.}

The hardening exponent is set in 100 300MPa, and the curves of minimum bending radius vs strengthen factor are shown in Fig.4.

It can be found that the minimum bending radius increases sharply with the strengthen factor from 100 to 300MPa. The minimum bending radius with strengthen factor $300 \mathrm{MPa}$ is twice as the corresponding value with strengthen $100 \mathrm{MPa}$. The bigger strengthen factor induces bigger yield stress, and the forming energy will be larger subsequently. The force exerted by dies need be larger, and the resistance to cross-section distortion of rectangular tube will be weaker. Therefore, the cross-section distortion is larger, and the tolerant deformation interval is narrow.

\subsubsection{The effects of hardening exponent.}

The strengthen factor is set in the 006-0.35, and the curves of minimum bending radius vs hardening exponent are shown in Fig.5.

The curves of minimum bending radius vs hardening exponent show that the minimum bending radius decreases from 50 to $30 \mathrm{~mm}$ with the hardening exponent from 0.05 to 0.35 . In the forming process, the work hardening effect will be more significant with bigger hardening exponent, and the the forming zone will transferred to the zone which is more difficult to deform. From macro view, the deformation of rectangular tube will be steady and uniform. Therefore, the rectangular tube with larger hardening exponent can be bent to smaller radius, and the forming limited interval is extended.

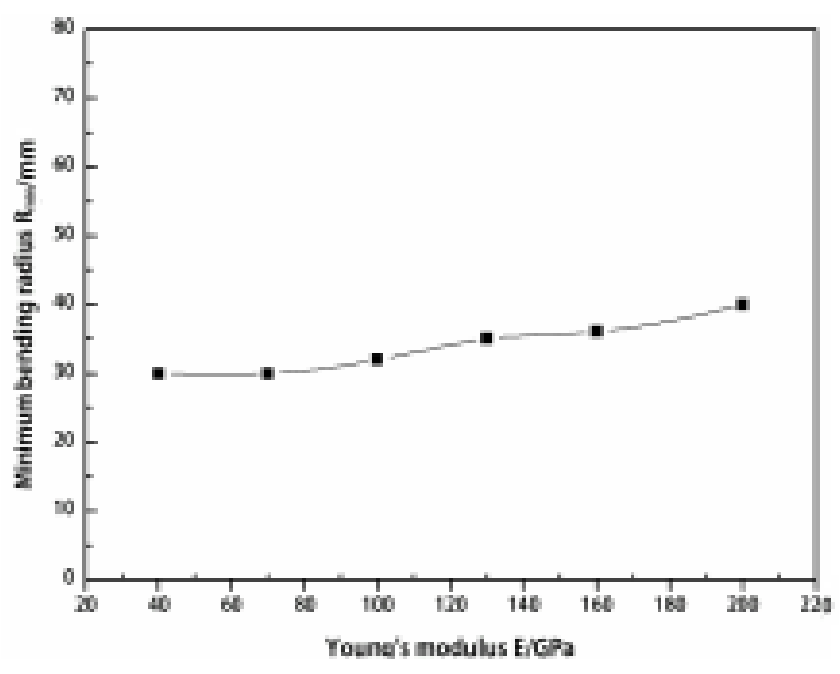

Fig.3 Curve of $R_{\min v s .} E$ 


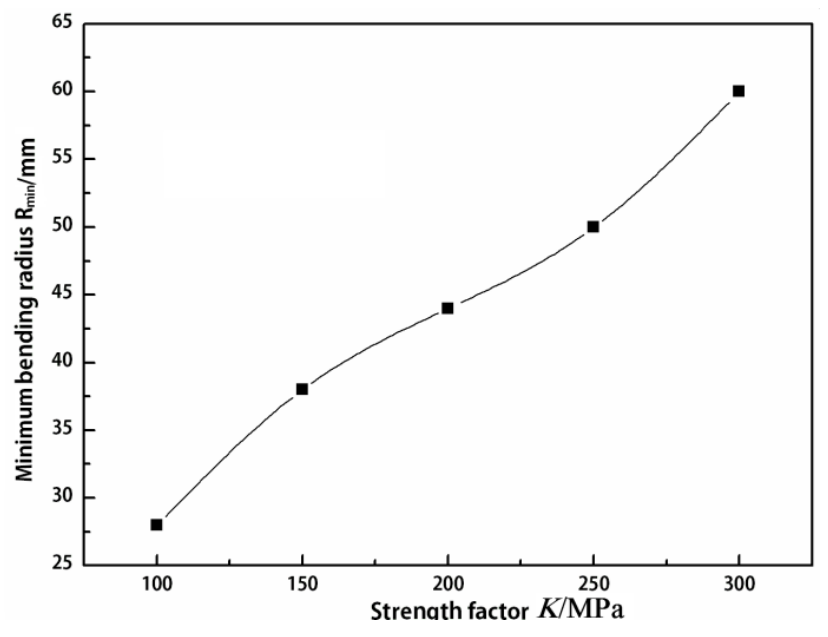

Fig.4Curves of $R_{\min V s .} K$

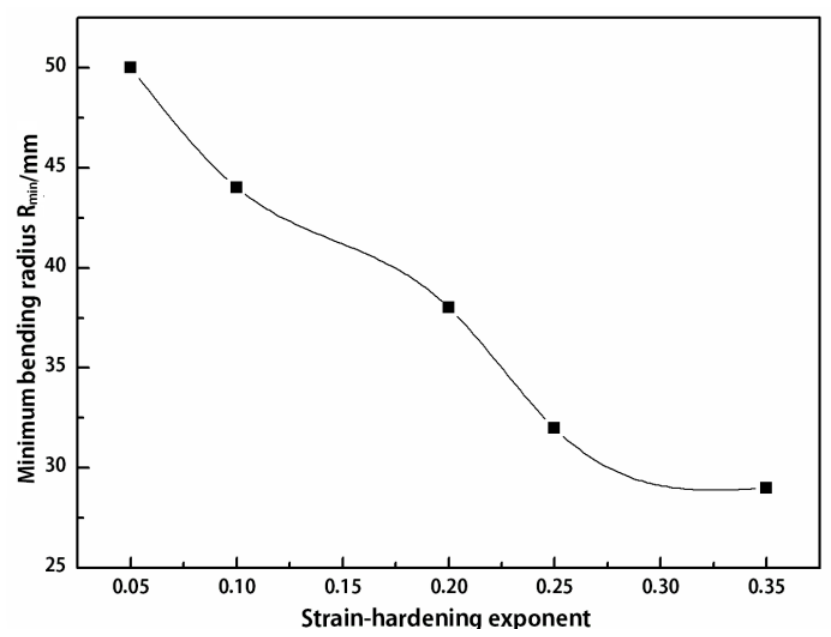

Fig5 Curves of $R_{\min V s .} n$

\subsection{The effects of dimensional parameters on forming limit}

\subsubsection{The effects of height.}

The height is set 6,12,24 and $48 \mathrm{~mm}$, and the distribution curves of minimum bending radius vs height are shown in Fig.6.

In Fig.12, the minimum bending radius increases in index type with the increase of height from 6 to $50 \mathrm{~mm}$. The height of rectangular tube increases the instability, and decreases the resistance to deform. With the increase of height, the constraining effects of webs on flanges are diminished, and the deformation of rectangular tube are enlarged. Therefore, the crosssection distortion is prone to enlarge. The forming limited interval will be shrank with increase of height.

\subsubsection{The effects of width.}

The width is set as 6,12 and $24 \mathrm{~mm}$, and the distribution curves of minimum bending radius vs width are shown in Fig.7.

It can be observed that the minimum bending radius decreases significantly with the width from 6 to $24 \mathrm{~mm}$. Width can increase the stability, and the core and mandrel can support the rectangular tube in larger zone. The flanges can be seen as plates constrained by core and mandrel, and the deformation will be uniform. Therefore, the cross-section distortion will be smaller, and the torrent forming limit interval will be expanded.

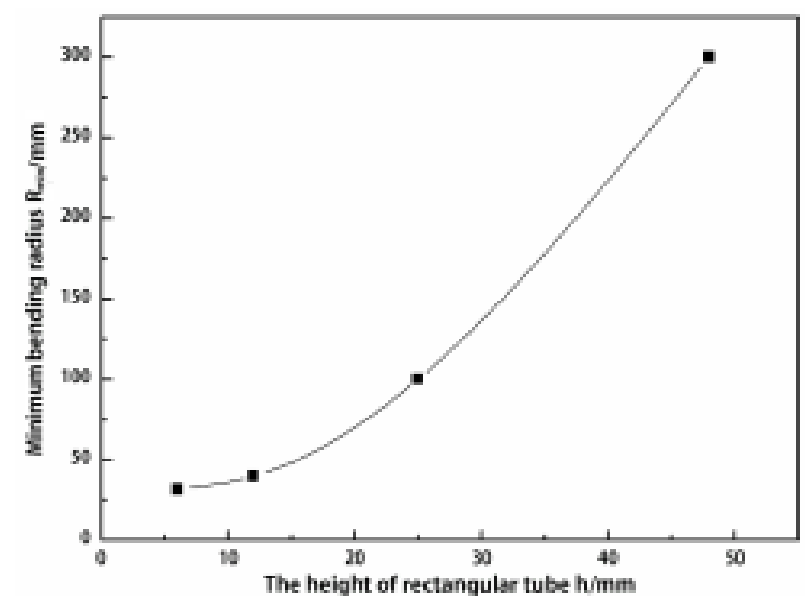

Fig.6 Curves of $R_{\min V s .} h$

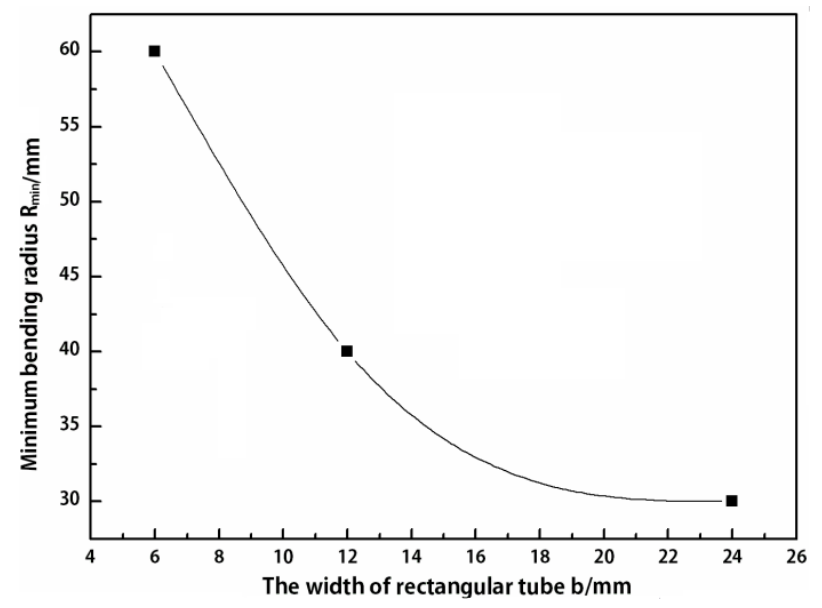

Fig.7 Curves of $R_{\min } \mathrm{Vs} . b$

\subsection{The effects of clearances between dies and tube on forming limit}

\subsubsection{The effects of clearance between tube and core die.}

The clearance is set as $0 \sim 2 \mathrm{~mm}$, and the distribution curves of minimum bending radius vs clearance between tube and core die are shown in Fig.8.

It can be observed that, the minimum bending radius has an astounding increase with clearance between mandrel and core from 0 to $2.0 \mathrm{~mm}$, and the forming limited intervals are narrow. In the bending process, the mandrel and core support the rectangular tube internally to resist the cross-section distortion. With the increase of the clearance, the mandrel and core cannot support the tube fully, and the flanges will collapse easier. Therefore, the crosssection distortion will increase. 


\subsubsection{The effects of clearance between tube and pressure die.}

The clearance is set as $0 \sim 2 \mathrm{~mm}$, and the distribution curves of minimum bending radius vs clearance between tube and pressure die are shown in Fig.9.

It can be found that the relevance between $R_{\min V S}$ $\mathrm{C}_{\mathrm{p}}$.is significant. The minimum bending radius increases from 28 to $75 \mathrm{~mm}$ with the clearance between tube and pressure die from 0 to $2.0 \mathrm{~mm}$. The pressure die constrains the rectangular tube externally, and the friction force exerted by pressure die will reduce with bigger clearance. The constraint of pressure die on rectangular tube will disappear, and the cross-section distortion will increase subsequently.

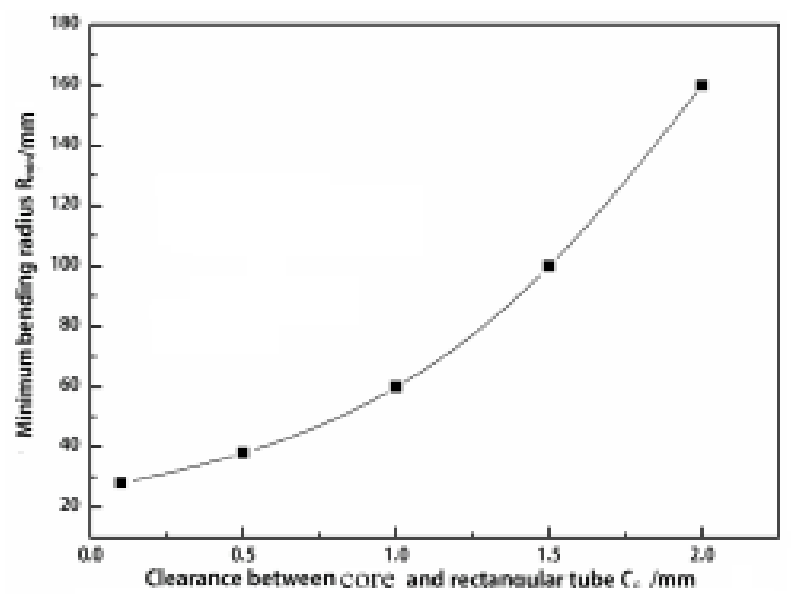

Fig.8 Curves of $R_{\min } \mathrm{Vs} \mathrm{C}_{\mathrm{c}}$

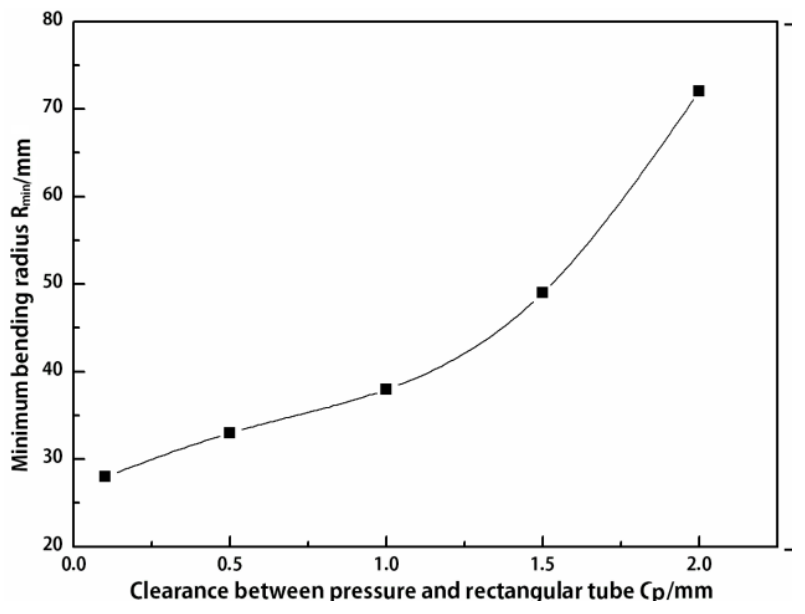

Fig.9 Curves of $R_{\min }$ Ss $\mathrm{C}_{\mathrm{p}}$

2.5 The effects of friction between dies and tube on forming limit

\subsubsection{The effects of friction between tube and core die.}

The friction is set as $0.06 \sim 0.3$, and the distribution curves of minimum bending radius vs friction between tube and core die are shown in Fig.10.

From Fig.10, the effects of friction between core and tube on minimum bending radius are significant.
The bigger the friction coefficients, the bigger the minimum bending radius. The friction force between core and tube are tangential, and it prevents the tube from feeding into the bent zone. Therefore, the material will gather backward after the tangential point, and the cross-section distortion will increase in bent zone. In practice, the cores will be lubricated by aviation oil to make the friction as small as possible.

\subsubsection{The effects of friction between tube and pres- sure die.}

The friction is set as $0.06 \sim 0.3$, and the distribution curves of minimum bending radius vs friction between tube and pressure die are shown in Fig.11.

It can be seen that the minimum bending radius keep constant almost with the increase of friction coefficient between pressure and tube. The pressure die constrains the tube after the tangential point, and it provides boost force to feed the material into the bent zone. Therefore, the friction force should be large, and in practice the interface between pressure and tube should be set as dry friction with no lubrication.

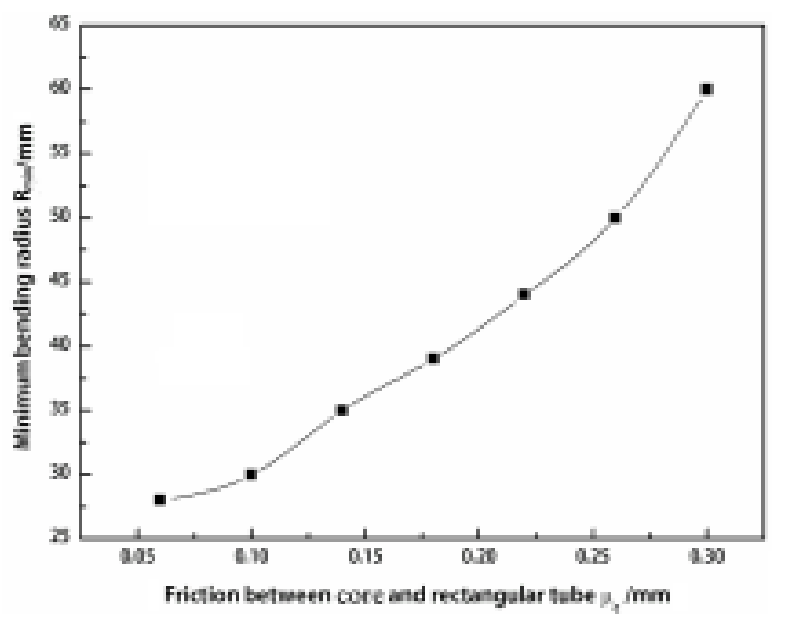

Fig.10 Curves of $R_{\min } \mathrm{VS} \mu_{\mathrm{c}}$

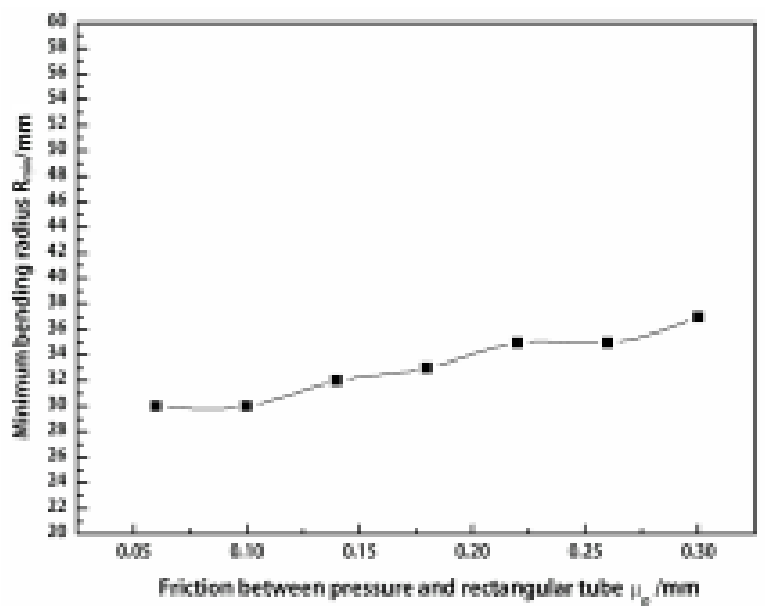

Fig.11 Curves of $R_{\min V s} \mu p$ 


\section{THE ESTABLISHMENT OF CROSS-SECTION DISTORTION LIMITED DIAGRAMS}

According to the study, the forming limited diagrams under different conditions are established as shown in Fig.12. When $\delta h>10 \%$, the cross-section distortion exceeds the allowable value, and the bending radius should be larger. When $\delta h=10 \%$, the cross-section distortion equals the allowable value, and the bending radius is the minimum bending radius. When $\delta h<10 \%$, the cross-section distortion is less than the allowable value, and the bending radius can be decreases further. The minimum bending radius can be determined based on geometrical dimensions, clearances, friction parameters and material parameters from Fig.12(a)-(d) respectively.
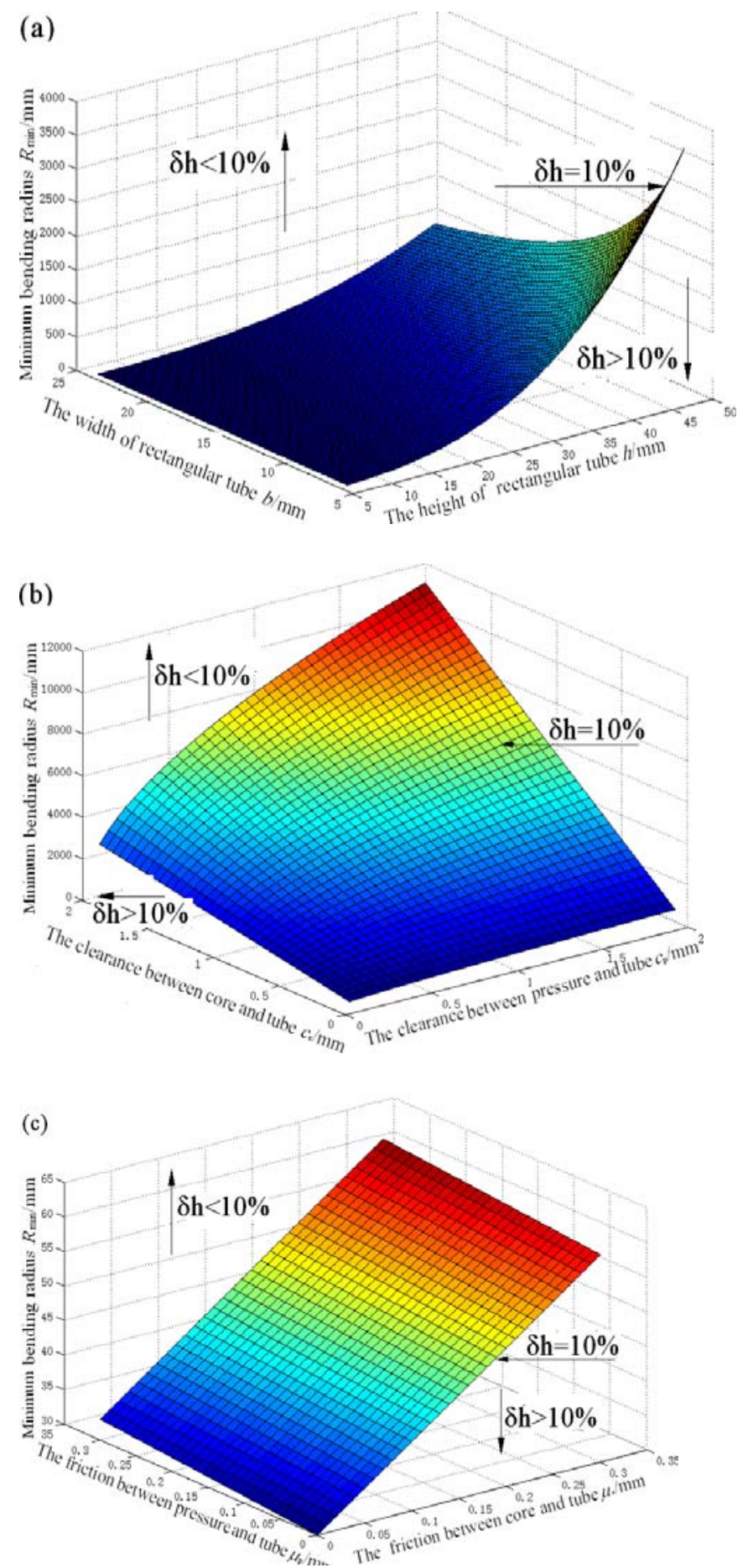

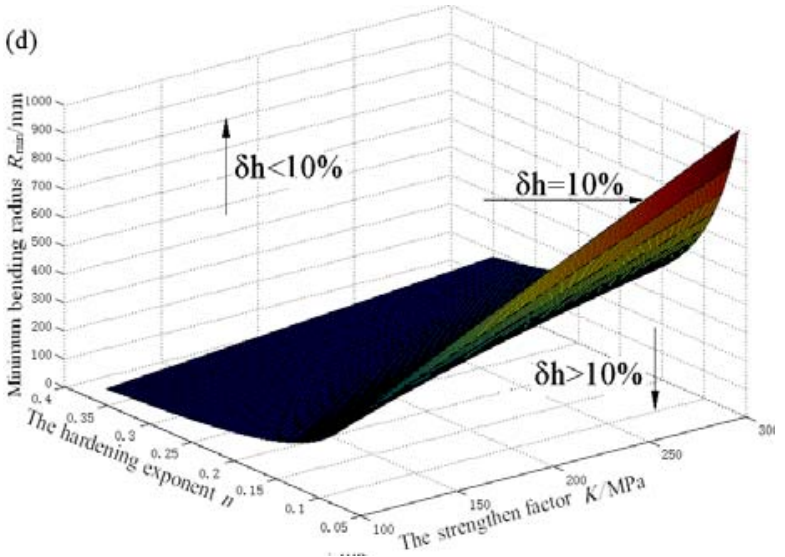

Fig.12 Cross-section distortion limited diagram: (a) based on geometrical dimensions; (b) based on clearances; (c) based on friction parameters; (d) based on material parameters

\section{CONCLUSIONS}

(1) The minimum bending radius when the crosssection distortion does not exceed $10 \%$ is defined as forming limit.

(2) With the increase of the strengthen factor, the height, clearance between mandrel and tube, clearance between pressure and tube, the friction between core and tube, the minimum bend radius increases and the bending limit decreases; with the increase of width and strain-hardening exponent, the minimum bending radius decreases and the bending limit increases.

(3) The 3D forming limit diagrams for cross-section distortion are obtained under different process parameters.

\section{SUMMARY}

The authors would like to thank Programs Supported by Ningbo Natural Science Foundation (2015A610096) and China Postdoctoral Science Foundation funded project (2015M571902) for the support given to the research.

\section{REFERENCES}

[1] H. Yang, M. Zhan, Y.L. Liu. Some advanced plastic processing technologies and their numerical simulation. J. Mater. Process. Technol. 151(2004) 63-69

[2] M. Hashmi. Aspects of tube and pipe manufacturing processes: Meter to nanometer diameter. J. Mater. Process. Technol. 179(2006)5-10

[3] H. Li, H. Yang, J. Yan, M. Zhan. Numerical study on deformation behaviors of thin-walled tube NC bending with large diameter and small bending radius. Comput. Mater. Sci. 45(2009) 921-934

[4] G.Y. Zhao, Y.L. Liu, H. Yang, C.H. Lu, R.J. Gu. Threedimensional finite-elements modeling and simulation of ro- 
tary-draw bending process for thin-walled rectangular tube. Mater Sci and Eng A. 499(2009)257-261

[5] G.Y. Zhao, Y.L. Liu, H. Yang. Effect of clearance on wrinkling of thin-walled rectangular tube in rotary-draw bending process. Int J of Adv Manuf Tech. 50(2010)85-92

[6] H. Yang, H. Li, M. Zhan. Friction role in bending behaviors of thin-walled tube in rotary-draw-bending under small bending radius. J. Mater. Process. Technol. 210(2010) 22732284

[7] H. Yang, Y. Lin. Wrinkling analysis for forming limit of tube bending process. J. Mater. Process. Tech. 152(2004) 363-369

[8] H. Li, H. Yang, M. Zhan, et al. A new method to accurately obtain wrinkling limit diagram in NC bending process of thin-walled tube with large diameter under different loading paths. J. Mater. Process. Tech. 177(2006)192-196

[9] G.Y. Zhao. The forming limit of wrinkling of rectangular tube in rortary draw bending. PHD. (2010) 133-134

[10] J. Yan, H. Yang, M. Zhan, et al. Forming limits under multi-index constraints in NC bending of aluminum alloy thin-walled tubes with large diameters. Sci. China Tech. Sci. 53(2010)326-342

[11] H. Li, H. Yang, M. Zhan. A study on critical thinning in thin-walled tube bending of al-alloy $5052 \mathrm{O}$ via coupled ductile fracture criteria. Proceedings of the 10th International Conference Numiform. Pohang, Korea, 2010, pp, 1286-1294

[12] Y. Okude, S. Sakaki, S. Yoshihara. Improving working limit in draw bending process of aluminum square tubes in large bending radius. Adv. Tech. Plasticity 2008, pp, 564569 